\title{
Commentary Cell death in sepsis: a matter of how, when, and where
} Heike Bantel ${ }^{1}$ and Klaus Schulze-Osthoff ${ }^{2}$

\author{
${ }^{1}$ Clinic of Gastroenterology, Hepatology and Endocrinology, Hannover Medical School, Carl-Neuberg-Strasse 1, D-30625 Hannover, Germany \\ ${ }^{2}$ Interfaculty Institute for Biochemistry, University of Tübingen, Hoppe-Seyler-Str. 4, D-72076 Tübingen, Germany
}

Corresponding author: Heike Bantel, bantel.heike@mh-hannover.de

Published: 31 July 2009

This article is online at http://ccforum.com/content/13/4/173

(c) 2009 BioMed Central Ltd

See related research by Hofer et al., http://ccforum.com/content/13/3/R93
Critical Care 2009, 13:173 (doi:10.1186/cc7966)

\begin{abstract}
Dysregulated cell death in several tissues is intimately involved in the pathogenesis of sepsis and contributes to multiple organ failure. Whether cell death during sepsis occurs by necrosis or apoptosis may depend on the cell type as well as the disease stage and is therefore a matter of intense debate. While lymphocyte apoptosis contributes to immunosuppression in sepsis, recent evidence suggests that necrosis of hepatocytes predominates in septic patients with liver dysfunction and correlates with poor survival. These distinct modes of cell death might have different consequences for the inflammatory response but are also critical for therapeutic interventions and the disease outcome. Understanding the complexity of death processes employing recently available serum biomarkers of cell death could lead to novel therapeutic approaches and assist in the steering of sepsis treatment.
\end{abstract}

Sepsis is the leading cause of morbidity and mortality in critically ill patients in many intensive care units. The pathophysiology of organ failure and death in patients with sepsis remains elusive. Previously, it was generally thought that sepsis represented an unbridled immune response with excessive cytokine production. The failure of almost all antiinflammatory agents to improve survival and the finding that the initial hyper-inflammatory response in sepsis is quickly followed by an immunocompromised state have considerably challenged this concept. Increasing evidence now suggests that extensive apoptotic death results in immune cell depletion and may compromise the ability of the patient to eradicate infections. Consequently, there is great hope that novel therapeutic approaches, including pharmacological agents that block apoptosis, will prove to be beneficial in sepsis treatment.

A study published in the previous issue of Critical Care, however, suggests that a general role of apoptosis in sepsis might be overestimated and that in other cell types or disease stages necrosis predominates [1]. Hofer and colleagues evaluated the use of two novel non-invasive biomarkers of cell death, caspase-cleaved cytokeratin-18 (CK-18) and uncleaved CK-18, for the prediction of clinical outcome in sepsis. Caspases, the key enzymes of apoptosis, cleave the intermediate filament protein CK-18 into specific fragments that are released into circulating blood and can be detected by a specific enzyme-linked immunosorbent assay (ELISA). Previous studies have demonstrated that this technique is a very sensitive biomarker for liver damage [2,3]. Moreover, when this assay is combined with a second ELISA that detects the total release of caspase-cleaved and uncleaved CK-18, even different forms of cell death, such as necrosis and apoptosis, can be discriminated [4]. Using these serological assays, Hofer and colleagues [1] found that patients with severe sepsis revealed higher levels of the cell death markers compared with postoperative patients or healthy individuals. In addition, serum CK-18 levels were significantly elevated in patients not surviving sepsis compared with survivors. Further analysis showed no differences of the biomarkers between survivors and non-survivors when liver function was retained. Importantly, however, significantly higher levels of total CK-18, indicative of necrosis, were observed in the nonsurviving group with decreased liver function. The results therefore suggest that, unlike apoptosis, hepatocyte necrosis is an early predictor of disease outcome in septic patients with liver dysfunction.

The relative contribution of apoptosis or necrosis to organ dysfunction in sepsis and most other diseases is unknown [5]. Necrosis is typically the consequence of acute metabolic perturbation with ATP depletion as it occurs in ischemia/ reperfusion and acute liver failure. Apoptosis, in contrast, represents the execution of an ATP-dependent death program that is often initiated by death receptor ligation, leading

CK-18 = cytokeratin-18; ELISA = enzyme-linked immunosorbent assay; IFN- $\gamma=$ interferon-gamma; IL = interleukin; PARP = poly(ADP-ribose) polymerase. 
to a caspase activation cascade [6]. Another important distinguishing feature of apoptotic versus necrotic cell death relates to inflammation. When the necrotic cell ruptures, an intense inflammatory response follows, owing to the release of intracellular contents. In contrast, inflammation is not typical of apoptosis, because phagocytic cells rapidly engulf apoptotic cells and thereby prevent the release of noxious intracellular compounds. Although apoptosis and necrosis have been considered distinct and independent phenomena, the two modes of cell death frequently coexist in different pathologies. A more extreme injury results in necrotic killing, whereas a milder injury triggers apoptosis. Understanding the processes leading to either apoptosis or necrosis is important for the development of effective interventions to prevent organ failure in sepsis.

During sepsis, there is extensive apoptotic death of lymphocytes and gastrointestinal epithelial cells [7]. The increased apoptotic death of lymphocytes is likely to be an important cause of the profound immunosuppression that is a hallmark of patients with sepsis. It has been suggested that apoptosis contributes to immunosuppression in prolonged sepsis in at least two major fashions: the elimination of key effector cells and, alternatively, the capacity of apoptotic cells to induce anergy and a shift to a $\mathrm{Th}_{2}$-cell response. Furthermore, apoptosis of gastrointestinal epithelial cells may compromise the integrity of the bowel wall, resulting in the translocation of bacteria or endotoxins into the systemic circulation. A contribution of apoptosis in sepsis is strongly supported by analysis of postmortem biopsies of septic patients, which revealed extensive apoptosis of lymphocytes and gastrointestinal epithelial cells [8].

The potential importance of apoptosis in the pathogenesis of sepsis is also illustrated by results from animal models that demonstrate that blocking lymphocyte apoptosis using caspase inhibitors improves survival in sepsis [7]. Nevertheless, these promising findings must be tempered by the new study showing that multiple pathways might be activated in dying cells during sepsis [1]. The simple inhibition of a particular cell death route therefore might not result in survival but rather lead to alternative pathways of cell death. Indeed, apoptosis may even be protective, by obviating the potential of injured cells to necrosis and further propagating the inflammatory response. In addition, a recent study indicated that inhibition of caspases could trigger a hyper-acute tumor necrosis factor (TNF)-induced shock in certain situations and actually exacerbate liver injury [9].

In addition to apoptosis, certain caspases, such as caspase-1, are involved in cytokine maturation by proteolytically processing the inactive precursors of interleukin (IL)-1 $\beta$ and IL-18, also known as interferon-gamma (IFN- $\gamma$ )-inducing factor, to the active cytokines. There are several independent observations that the generation of cytokines by proinflammatory caspases is indeed required for survival in sepsis [10]. In support of this hypothesis, recombinant IFN- $\gamma$ does not impair sepsis survival in clinical trials but seems to improve it [11]. Inhibition of caspases therefore could further dampen the immune response and exacerbate immunosuppression. Thus, despite some encouraging results in animal studies, there are potential pitfalls that could preclude the simple use of caspase inhibitors for sepsis treatment.

The finding by Hofer and colleagues [1] that necrosis but not apoptosis is the primary mode of hepatocyte death further argues against a therapeutic efficacy of caspase inhibitors, at least in septic patients with liver dysfunction. Focal necrosis in hepatocytes in the central vein region has previously been detected in autopsies of septic patients [8]. The hepatocyte necrosis is presumably caused by hypoxia, mitochondrial dysfunction, and decreased ATP levels. In line with this, Hofer and colleagues [1] show that the increased necrosis correlates with high lactate levels that are characteristic for acute liver failure. It is interesting to note that high-mobilitygroup box protein-1 (HMGB1), which is specifically released from necrotic but not apoptotic cells, is systemically elevated in human sepsis and functions as a mediator of endotoxin lethality in mice [12].

Intense research has recently delineated the signaling pathway of necrotic cell death. Necrosis has long been considered an uncontrolled mode of cell death, but it has become clear that necrosis is a regulated event controlled by several enzymes such as the serine/threonine kinase RIP1 and the DNA repair enzyme poly(ADP-ribose) polymerase (PARP) [13]. Necrosis is often associated with mitochondrial dysfunction, oxidative stress, and disturbance of calcium homeostasis. Consequently, there are many potential approaches to pharmacologically modulate necrosis, including inhibition of the formation of reactive oxygen species or inhibition of the influx of extracellular calcium. Moreover, septic mice that are deficient in PARP have improved survival, and administration of a PARP inhibitor is beneficial in animal models [14]. Finally, recently designed inhibitors of RIP1, called necrostatins, represent new cytoprotective agents that might impair necrotic tissue damage in sepsis [15]. Certainly, further work is needed to evaluate future approaches for modulating cell death in clinical applications of sepsis treatment.

\section{Competing interests}

The authors declare that they have no competing interests.

\section{References}

1. Hofer S, Brenner T, Bopp C, Steppan J, Lichtenstern C, Weitz J, Bruckner T, Martin E, Hoffmann U, Weigand MA: Cell death serum biomarkers are early predictors for survival in severe septic patients with hepatic dysfunction. Crit Care 2009, 13: R93.

2. Bantel $H$, Lügering $A$, Heidemann J, Volkmann X, Poremba C, Strassburg CP, Manns MP, Schulze-Osthoff K: Detection of apoptotic caspase activation in sera from patients with chronic HCV infection is associated with fibrotic liver injury. Hepatology 2004, 40:1078-1087. 
3. Seidel $N$, Volkmann $X$, Länger $F$, Flemming $P$, Manns MP, Schulze-Osthoff $\mathrm{K}$, Bantel $\mathrm{H}$ : The extent of liver steatosis in chronic hepatitis $\mathrm{C}$ virus infection is mirrored by caspase activity in serum. Hepatology 2005, 42:113-120.

4. Volkmann X, Anstaett M, Hadem J, Stiefel P, Bahr MJ, Lehner F, Manns MP, Schulze-Osthoff $\mathrm{K}$, Bantel $\mathrm{H}$ : Caspase activation is associated with spontaneous recovery from acute liver failure. Hepatology 2008, 47:1624-1633.

5. Fischer U, Schulze-Osthoff K: New approaches and therapeutics targeting apoptosis in disease. Pharmacol Rev 2005, 57: 187-215.

6. Leist $M$, Jäättelä $M$ : Four deaths and a funeral: from caspases to alternative mechanisms. Nat Rev Mol Cell Biol 2001, 2:589598.

7. Hotchkiss RS, Nicholson DW: Apoptosis and caspases regulate death and inflammation in sepsis. Nat Rev Immunol 2006, 6:813-822.

8. Hotchkiss RS, Swanson PE, Freeman BD, Tinsley KW, Cobb JP, Matuschak GM, Buchman TG, Karl IE: Apoptotic cell death in patients with sepsis, shock, and multiple organ dysfunction. Crit Care Med 1999, 27:1230-1251.

9. Cauwels A, Janssen B, Waeytens A, Cuvelier C, Brouckaert P. Caspase inhibition causes hyperacute tumor necrosis factorinduced shock via oxidative stress and phospholipase A2. Nat Immunol 2003, 4:387-393.

10. Scott AM, Saleh M: The inflammatory caspases: guardians against infections and sepsis. Cell Death Differ 2007, 14:2331.

11. Döcke WD, Randow F, Syrbe U, Krausch D, Asadullah K, Reinke P, Volk HD, Kox W: Monocyte deactivation in septic patients: restoration by IFN-gamma treatment. Nat Med 1997, 3:678681.

12. Fink MP: Bench-to-bedside review: high-mobility group box 1 and critical illness. Crit Care 2007, 11:229.

13. Hitomi J, Christofferson DE, Ng A, Yao J, Degterev A, Xavier RJ, Yuan J: Identification of a molecular signaling network that regulates a cellular necrotic cell death pathway. Cell 2008, 135:1311-1323.

14. Soriano FG, Liaudet L, Szabó E, Virág L, Mabley JG, Pacher P, Szabó C: Resistance to acute septic peritonitis in poly(ADPribose) polymerase-1-deficient mice. Shock 2002, 17:286292.

15. Vandenabeele P, Declercq W, Berghe TV: Necrotic cell death and 'necrostatins': now we can control cellular explosion. Trends Biochem Sci 2008, 33:352-355. 